\title{
Oligodendrocyte Regeneration and CNS Remyelination Require TACE/ADAM17
}

\author{
- Davier Palazuelos, ${ }^{1}$ Michael Klingener, ${ }^{1}$ Elaine W. Raines, ${ }^{2}$ Howard C. Crawford, ${ }^{3}$ and Adan Aguirre ${ }^{1}$ \\ ${ }^{1}$ Department of Pharmacological Sciences, Centers for Molecular Medicine, Stony Brook University, SUNY, Stony Brook, New York 11794, ${ }^{2}$ Department of \\ Pathology, University of Washington, Seattle, Washington 98195, and ${ }^{3}$ Department of Molecular and Integrative Physiology, University of Michigan, Ann \\ Arbor, Michigan 48109
}

\begin{abstract}
The identification of the molecular network that supports oligodendrocyte $(\mathrm{OL})$ regeneration under demyelinating conditions has been a primary goal for regenerative medicine in demyelinating disorders. We recently described an essential function for TACE/ADAM17 in regulating oligodendrogenesis during postnatal myelination, but it is unknown whether this protein also plays a role in OL regeneration and remyelination under demyelinating conditions. By using genetic mouse models to achieve selective gain- or loss-of-function of TACE or EGFR in OL lineage cells in vivo, we found that TACE is critical for EGFR activation in OLs following demyelination, and therefore, for sustaining OL regeneration and CNS remyelination. TACE deficiency in oligodendrocyte progenitor cells following demyelination disturbs $\mathrm{OL}$ lineage cell expansion and survival, leading to a delay in the remyelination process. EGFR overexpression in TACE deficient OLs in vivo restores OL development and postnatal CNS myelination, but also OL regeneration and CNS remyelination following demyelination. Our study reveals an essential function of TACE in supporting OL regeneration and CNS remyelination that may contribute to the design of new strategies for therapeutic intervention in demyelinating disorders by promoting oligodendrocyte regeneration and myelin repair.
\end{abstract}

Key words: ADAM17; EGFR; oligodendrocyte precursor; remyelination; TACE

Significance Statement

Oligodendrocyte (OL) regeneration has emerged as a promising new approach for the treatment of demyelinating disorders. By using genetic mouse models to selectively delete TACE expression in oligodendrocyte progenitors cells (OPs), we found that TACE/ADAM17 is required for supporting OL regeneration following demyelination. TACE genetic depletion in OPs abrogates EGFR activation in OL lineage cells, and perturbs cell expansion and survival, blunting the process of CNS remyelination. Moreover, EGFR overexpression in TACE-deficient OPs in vivo overcomes the defects in OL development during postnatal development but also OL regeneration during CNS remyelination. Our study identifies TACE as an essential player in OL regeneration that may provide new insights in the development of new strategies for promoting myelin repair in demyelinating disorders.

\section{Introduction}

During the process of oligodendrocyte $(\mathrm{OL})$ regeneration and remyelination, oligodendrocyte progenitor cells (OPs) present in

Received Sept. 22, 2014; revised July 8, 2015; accepted July 20, 2015.

Author contributions: J.P. and A.A. designed research; J.P., M.K., and A.A. performed research; E.W.R. and H.C.C. contributed unpublished reagents/analytic tools; J.P., M.K., and A.A. analyzed data; J.P. and A.A. wrote the paper.

This work was supported by National Institutes of Health 4R00NS057944-03 (A.A.) and 1R01MH099384-01 (A.A.), and the National Multiple Sclerosis Society (J.P.). We thank Dr Ratner (Cancer and Blood Diseases Institute, Cincinnati Children's Hospital Research Foundation) for the CNP-hEGFR mouse line; S. Van Horn from the Central Microscopy Imaging Center at SUNY for her assistance in the electron microscopy analysis; H. Colognato, S. Tsirka, and M. Frohman for critically reading this paper; and to all our colleagues at the Pharmacology Department at SUNY, Stony Brook, for their support.

The authors declare no competing financial interests.

Correspondence should be addressed to either Dr Adan Aguirre or Dr Javier Palazuelos, Centers for Molecular

Medicine, Stony Brook University, SUNY, Stony Brook, NY 11794. E-mail: Adan.Aguirre@StonyBrook.edu or Javier.PalazuelosDiego@StonyBrook.edu.

DOI:10.1523/JNEUROSCI.3937-14.2015

Copyright $\odot 2015$ the authors $\quad 0270-6474 / 15 / 3512241-07 \$ 15.00 / 0$ demyelinating lesions undergo to a series of cellular events including proliferation, migration and differentiation to secure OL regeneration and fully repair the demyelinated lesion (Fancy et al., 2011). Therefore, the identification of the molecular network that governs these processes under demyelinating conditions represents an essential step for the design of cell-based strategies to promote OL regeneration, myelin repair, and functional recovery in demyelinating disorders (Huang et al., 2011).

ADAMs (a disintegrin and metalloproteinase proteins) represents a family of transmembrane proteins that control a variety of cell functions by modulating the ectodomain shedding of several membrane-anchored signaling molecules (Edwards et al., 2008; Weber and Saftig, 2012). In the context of CNS myelination, we recently described that ADAM17, also known as TNF $\alpha$ converting enzyme (TACE), is essential for OL development during postnatal myelination of the subcortical white matter (SCWM) by modulating OP expansion, cell cycle exit, and sur- 
vival (Palazuelos et al., 2014). TACE appears to be the main PMA-sensitive ADAM protein responsible for EGFR ligand shedding and EGFR signaling activation in OL lineage cells during SCWM myelination (Palazuelos et al., 2014), suggesting that it may be involved in OL regeneration and CNS remyelination. Moreover, it is widely accepted that EGFR signaling plays a crucial role in modulating OP expansion and OL survival during CNS myelination, but more importantly under pathological conditions (Aguirre et al., 2007; Ivkovic et al., 2008). EGFR hypofunction delays OL regeneration after focal-induced demyelination (Aguirre and Gallo, 2007), and EGFR signaling activation or overexpression enhances OP expansion and sustains OL lineage cell survival following postnatal hypoxia or focal demyelination (Aguirre et al., 2007; Scafidi et al., 2014). Although ADAM17 modulates numerous processes during development and homeostasis (Weber and Saftig, 2012), its relevance has been highlighted under pathological situations (Yang et al., 2006; Pruessmeyer and Ludwig, 2009; Scheller et al., 2011). However, a role for TACE during OL regeneration and CNS remyelination has never been addressed. Here, we show that TACE/ADAM17, at least in part, by modulating EGFR signaling activation in OL lineage cells, is essential for OL regeneration during CNS remyelination following demyelination.

\section{Materials and Methods}

Animals. All animal procedures were performed according to the Institutional Animal Care and Use Committee of Division of Laboratory Animal Resources, SUNY Stony Brook School of Medicine, and the National Institutes of Health Guide for the Care and Use of Laboratory Animals. The generation and characterization of NG2-dsRed (Zhu et al., 2008) and TACE ${ }^{\mathrm{fl} / \mathrm{fl}}$ (Peschon et al., 1998) mouse lines was performed as previously reported (Palazuelos et al., 2014). The NG2-Cre (Zhu et al., 2008), Wa2 (Luetteke et al., 1994; EgfrWa2), PDGFR $\alpha$-creERT2 (Rivers et al., 2008), and Rosa-YFP (Palazuelos et al., 2014) mice were obtained from The Jackson Laboratory. The CNP-hEGFR mouse line was kindly donated by Dr Ratner (Cancer and Blood Diseases Institute, Cincinnati Children's Hospital Research Foundation). All mouse strains used in this study were maintained in the $C 57 B L / 6$ genetic background. Males and females were used in this study. Cell proliferation was assessed by injecting 5-bromo-2'-deoxyuridine (BrdU) at $100 \mathrm{mg} / \mathrm{kg} 3 \mathrm{~h}$ before the end of the experiment (Palazuelos et al., 2014).

Cuprizone-induced demyelination. Eight to 12-week-old mice were fed ad libitum with $0.2 \%$ cuprizone (Cupz; cyclohexylidenehydrazide, Harlan) mixed into pelleted chow (diet TD.8604; Teklad Laboratories) for 9 weeks to induce demyelination (Klingener et al., 2014). Similar levels of demyelination were observed in all mouse lines analyzed in this study, with $20.1 \pm 4.5 \%$ of MBP protein levels and $15.5 \pm 3.4 \%$ of $\mathrm{CC}^{+}{ }^{+}$cells were found in the SCWM of 9 weeks Cupz-treated mice as compared with regular fed mice. Recombination was induced following demyelination in PDGFR $\alpha-\mathrm{Cre}^{\mathrm{tm}}: \mathrm{TACE}^{\mathrm{w} / \mathrm{w}}:$ Rosa-YFP (w/w) and PDGFR $\alpha-$ $\mathrm{Cre}^{\mathrm{tm}}: \mathrm{TACE}^{\mathrm{fl} / \mathrm{fl}}:$ Rosa-YFP mice (fl/fl) which received three consecutive injections of tamoxifen (Tam; $24 \mathrm{~h}$ apart; $60 \mathrm{mg} / \mathrm{kg}$ i.p., dissolved in sesame oil) starting the same day of Cupz diet removal. We did not observe major side effects in $\mathrm{w} / \mathrm{w}$ or $\mathrm{fl} / \mathrm{fl}$ mice. Western blot analysis of SCWM extracts at $24 \mathrm{~h}$ after the last Tam indicated a $40 \pm 2.5 \%$ reduction of TACE protein levels in the SCWM of PDGFR $\alpha$-Cre ${ }^{\text {tm }}:$ TACE $^{\mathrm{fl} / \mathrm{fl}}$ : Rosa-YFP mice as compared with PDGFR $\alpha-\mathrm{Cre}^{\mathrm{tm}}: \mathrm{TACE}^{\mathrm{w} / \mathrm{w}}:$ Rosa-YFP mice.

Immunofluorescence and microscopy. Free-floating coronal brain sections were blocked with $5 \%$ goat serum, and incubated with the indicated primary antibodies, followed by incubation with the appropriate mouse, rat or rabbit highly cross-adsorbed AlexaFluor-488, 547, or 647 secondary antibodies (Invitrogen). The following primary antibodies were used: anti-NG2 (AB_91789) anti-BrdU (AB_609568), anti-MBP (AB_510039) anti-CNP (AB_510037), anti-CC1 (AB_213434), anti-Caspase3 (AB_2069872), anti-pEGFR (AB_2096270), anti-YFP (AB_1196615), and anti-Adam17 (AB_302796). Confocal images were acquired using a Leica-TCS-SP5 microscope. SCWM analysis included the corpus callosum and cingulum, and a minimum of six correlative slices from a 1-in-10 series located between -1.0 and $+1.0 \mathrm{~mm}$ from bregma. Cell counting was performed by using a newCast optical fractionator (Visiopharm) and data are presented as the mean cell number per cubic millimeter $(\times 1000)$.

Electron microscopy. Mice were perfused intracardially with 2\% PFA/ $2.5 \%$ glutaraldehyde in $0.1 \mathrm{~m}$ PBS fixed overnight, and sectioned at 50 $\mu \mathrm{m}$, placed in $2 \%$ osmium tetroxide in $0.1 \mathrm{~m}$ phosphate and ethyl alcohols and vacuum infiltrated in Durcupan ACM embedding agent. Ultrathin sections $(70-80 \mathrm{~nm})$ were obtained using ultramicrotome and counterstained with uranyl acetate and lead citrate. Samples were analyzed with a Tecnai Spirit Bio-Twin G2transmission electron microscope (FEI) coupled to anAMTXR-60CCD Digital Camera System (Advanced Microscopy Techniques).

Human brain samples. Postmortem brain tissue samples from multiple sclerosis (MS) patients $(n=3)$ were provided by the Rocky Mountain MS Center at Anschutz Medical Campus, Colorado. MS tissue samples corresponded to subjects clinically and neuropathologically diagnosed with secondary progressive multiple sclerosis (SPMS), and included two females and one male, with mean age of $60 \pm 10$ and 65 years, respectively. Lesions were classified as normal appearing white matter (NAWM) according to the standard pathological practices at the Rocky Mountain MS Center. Snap frozen samples from three NAWM regions from each subject were cut into $40-\mu \mathrm{m}$-thick sections and fixed with $\mathrm{methanol} / \mathrm{acetone}$ for $10 \mathrm{~min}$. Autofluorescence was quenched by treatment with $1 \%$ Sudan black in $70 \%$ ethanol.

Western blot analysis. SCWM tissue was microdissected from 300- $\mu \mathrm{m}-$ thick coronal mouse brain sections and processed for RNA, protein extraction, or FAC-sorting. Proteins were extracted by using RIPA lysis buffer, separated on acrylamide gels and transferred to PVDF membranes. Antibody binding was detected using horseradish peroxidaseconjugated secondary antibodies (Santa Cruz Biotechnology) and an enhanced chemiluminescence system (Millipore).

FACS. FACS purification of NG2-dsRed ${ }^{+}$cells was performed as previously described (Aguirre et al., 2007). Cell suspensions were analyzed using a FAC-Star-plus instrument (Beckton Dickinson). Purified NG2$\mathrm{dsRed}^{+}$cells were processed for RNA isolation and qPCR analysis.

Real-time PCR. RNA extraction was performed using TRIzol (Invitrogen). Total RNA was reverse-transcribed using the SuperScript cDNA Synthesis kit (Invitrogen). Real-time quantitative PCR was performed with the SYBR Green system and run in a LightCycler 480 Real-time PCR system (Roche). Primers sequences for TACE are as follows: F:5'-GTAC GTCGATGCAGAGCAAA; R:5'-CATCCTCTGGTGGTCCAGTT.

Statistics. Results shown represent the mean \pm SEM, and the number of experiments is indicated in every case. Statistical analysis was performed by one- or two-way ANOVA as appropriate. A post hoc analysis was made by the Student Neuman-Keuls test.

\section{Results}

TACE is expressed by $O L$ lineage cells in the normal appearing white matter of MS subjects

We recently described an essential function for TACE in OL development during postnatal myelination. To determine whether TACE is also involved in OL regeneration, we first analyzed TACE expression in OL lineage cells in the NAWM of postmortem samples from SPMS subjects. Immunofluorescence (IF) analysis showed TACE expression in $\mathrm{OPs}\left(\mathrm{NG}^{+}{ }^{+}\right.$cells; Fig. $\left.1 A\right)$ and cells undergoing $\mathrm{OL}$ differentiation $\left(\mathrm{CNP}^{+}\right.$cells; Fig. $\left.1 B\right)$ in the NAWM of MS subjects, suggesting a role for TACE in OL regeneration.

\section{TACE is upregulated in OL lineage cells after Cupz-induced} demyelination

To investigate a role for TACE in OL regeneration and remyelination, we used the animal model of Cupz-induced global demyelination (Klingener et al., 2014). We first characterized TACE 
A

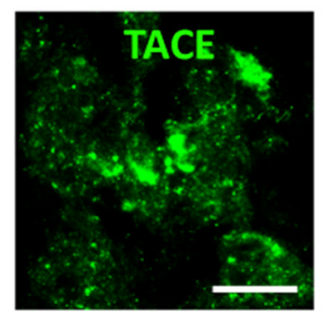

B

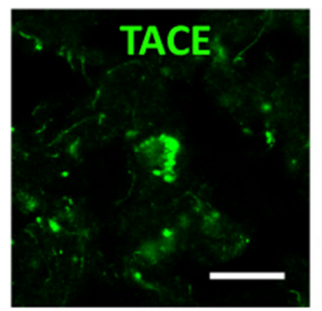

D

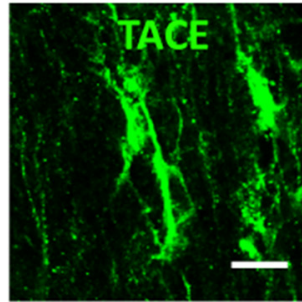

E

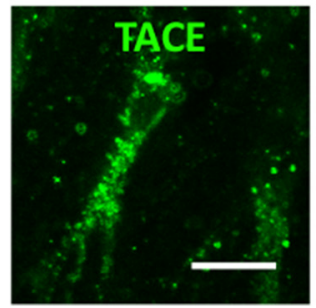

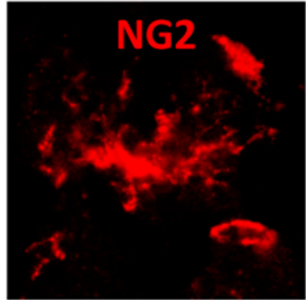
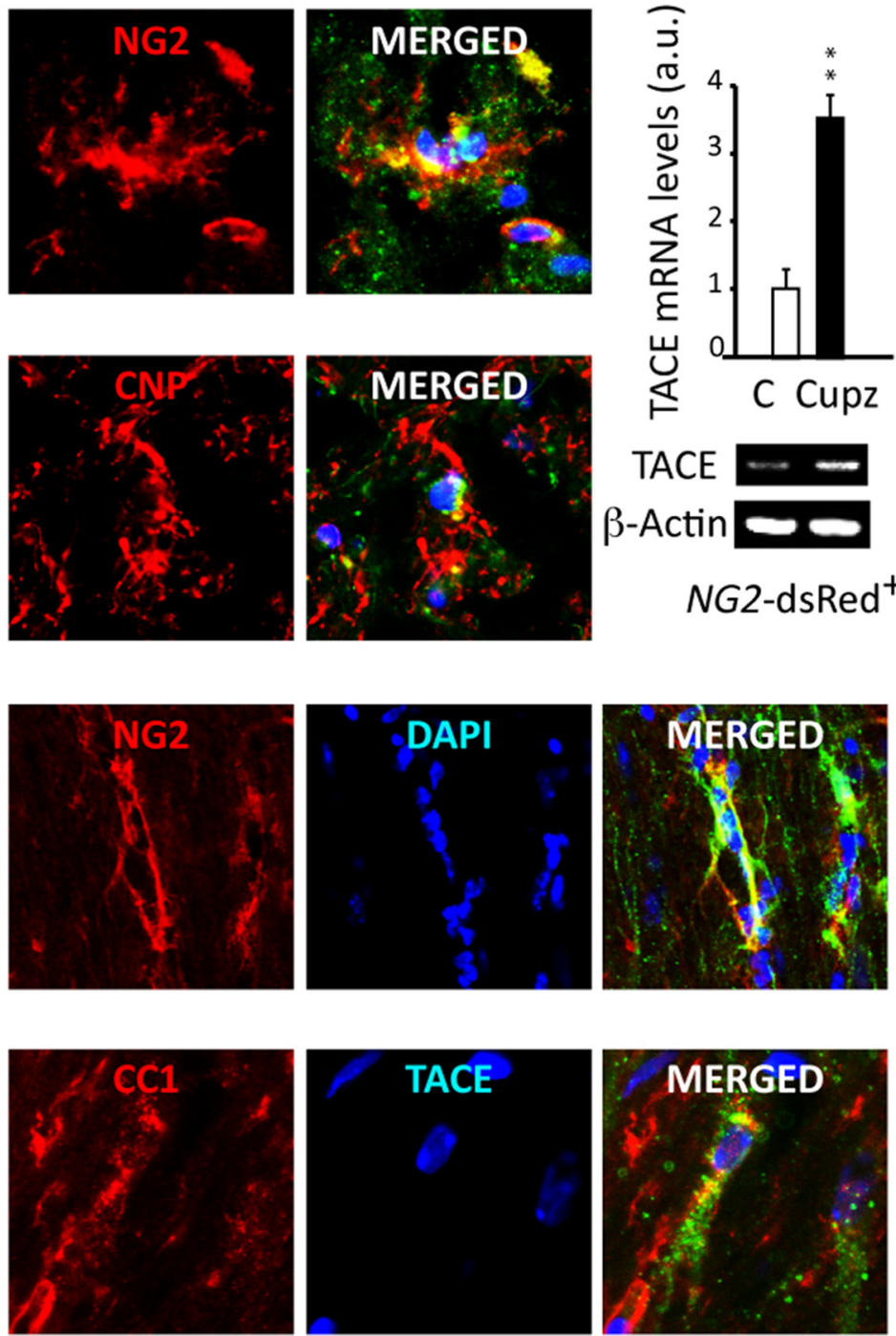

Figure 1. TACE is expressed by $0 \mathrm{~L}$ lineage cells in the white matter of subjects with MS and is upregulated following cuprizone-induced demyelination. $\boldsymbol{A}, \boldsymbol{B}$, Postmortem brain tissue samples from subjects with SPMS were used to characterize TACE expression in OL lineage cells in NAWM regions. Immunofluorescence analysis revealed TACE expression in OPs ( $\boldsymbol{A}$; NG2 ${ }^{+}$cells) and cells undergoing $0 \mathrm{~L}$ differentiation ( $\boldsymbol{B} ; \mathrm{CNP}^{+}$cells). $\boldsymbol{C}-\boldsymbol{E}$, TACE is upregulated in OL lineage cells in the SCWM following Cupz-induced demyelination. Adult NG2-dsRed mice were fed with Cupz or normal chow for 9 consecutive weeks. $\boldsymbol{C}, N G 2$-dsRed ${ }^{+}$FAC-sorted cells were obtained from the SCWM of mice fed with Cupz or normal diet for 9 weeks, and TACE mRNA levels were analyzed by qPCR. $\boldsymbol{D}$, $\boldsymbol{E}$, Immunofluorescence analysis in the mouse SCWM after 9 weeks of Cupz treatment revealed TACE expression in $0 \mathrm{Ps}\left(\boldsymbol{D} ; \mathrm{NG} 2^{+}\right)$and cells undergoing $0 \mathrm{~L}$ differentiation $\left(\boldsymbol{E} ; \mathrm{CC}^{+}{ }^{+}\right)$. Histograms are expressed as arbitrary units (a.u.) after $\beta$-actin normalization. Data from animal models are shown as mean \pm SEM $n=4-6$ brains for each time point. Scale bars, $10 \mu \mathrm{m}$. ${ }^{* *} p<0.01$ versus controls.

expression in OPs following demyelination. NG2-dsRed ${ }^{+}$cells were FAC-sorted from the SCWM of NG2-dsRed mice after 9 weeks of Cupz or control treatment. Real-time PCR analysis evidenced higher TACE mRNA levels in OPs from the SCWM of Cupz-treated mice compared with controls (Fig. 1C). Moreover, immunofluorescence analysis of SCWM sections of mice fed with Cupz for 9 weeks confirmed the high expression levels of TACE in $\mathrm{OPs}\left(\mathrm{NG}^{+}{ }^{+}\right.$; Fig. $\left.1 D\right)$ and OLs $\left(\mathrm{CC1}^{+}\right.$; Fig. $\left.1 E\right)$. Altogether, the upregulation of TACE in WM OL lineage cells observed following Cupz-induced demyelination and its presence in OL lineage cells in MS tissue samples suggest that this metalloprotease may be involved in OL regeneration and CNS remyelination.

\section{TACE depletion in OPs following demyelination delays}

CNS remyelination

To investigate TACE functions during OL regeneration and CNS remyelination, we used the Cupz model of demyelina- tion, and the PDGFR $\alpha-\mathrm{Cre}^{\mathrm{tm}}: \mathrm{TACE}^{\mathrm{fl} / \mathrm{fl}}:$ Rosa-YFP mouse line (Palazuelos et al., 2014), to specifically deplete TACE expression in OPs after tamoxifen (Tam) administration (Fig. 2A). $P D G F R \alpha-C_{r}{ }^{\mathrm{tm}}:$ TACE $^{\mathrm{w} / \mathrm{w}}:$ Rosa-YFP $(\mathrm{w} / \mathrm{w})$ and PDGFR $\alpha-$ $\mathrm{Cre}^{\mathrm{tm}}: \mathrm{TACE}^{\mathrm{fl} / \mathrm{fl}}:$ Rosa-YFP (fl/fl) mice were fed with Cupz for 9 weeks, and upon Cupz removal, Tam was administered for 3 consecutive days. Western blot (WB) analysis at 1 and 2 weeks after the last Tam administration evidenced a delay in the process of SCWM remyelination in $\mathrm{fl} / \mathrm{fl}$ mice, as we detected reduced myelin-related protein levels in SCWM extracts compared with w/w mice (Fig. $2 B, C$ ). We confirmed this result by immunohistochemistry analysis, as we detected greater areas of hypomyelination in the SCWM of PDGFR $\alpha-C r e^{\mathrm{tm}}$ : $\mathrm{TACE}^{\mathrm{fl} / \mathrm{fl}}$ :Rosa-YFP mice that correlated with reduced numbers of mature OLs ( $\mathrm{YFP}^{+}$cells; Fig. $2 D$ ) compared with w/w mice. Ultrastructural analysis by electron microscopy con- 


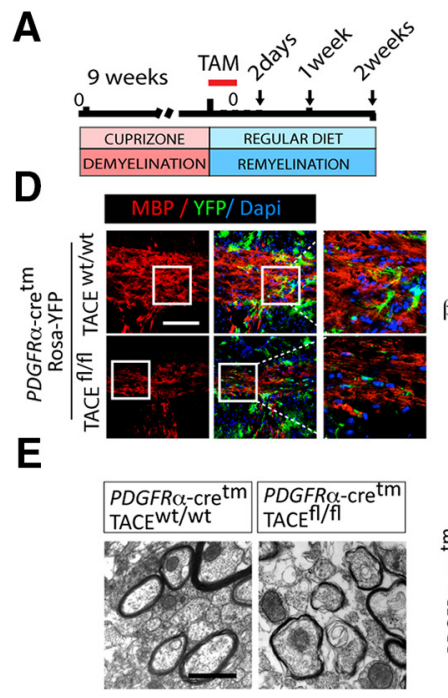

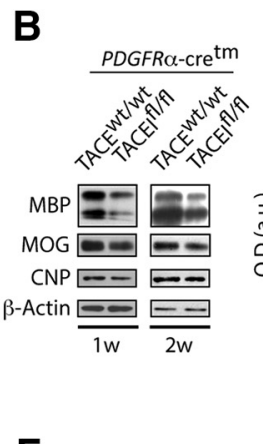

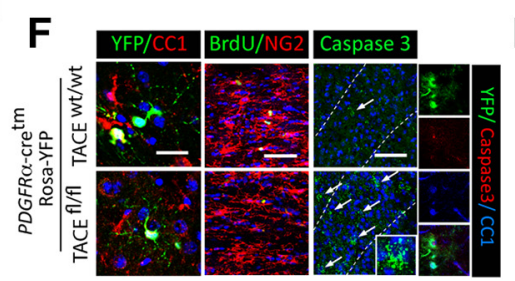

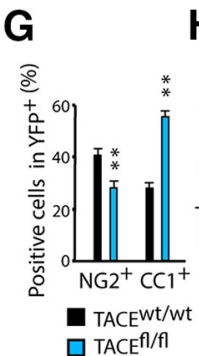
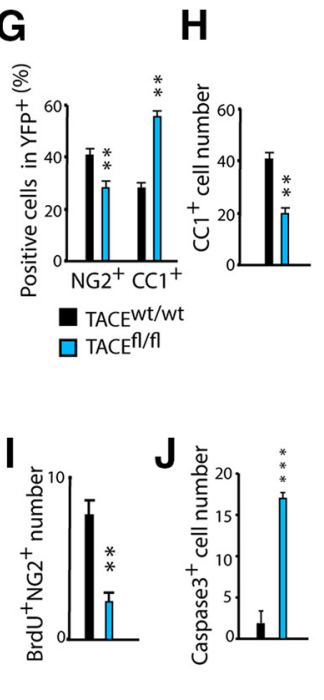

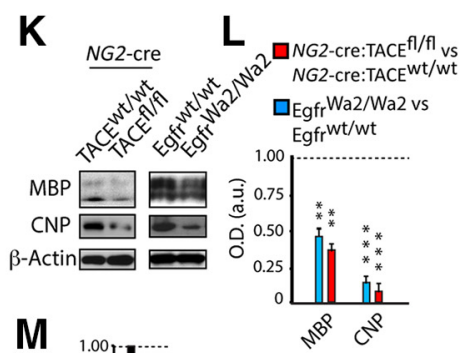

M
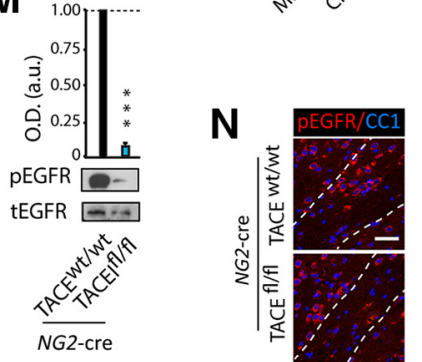

Figure 2. TACE genetic deletion in OPs following demyelination delays OL regeneration and CNS remyelination. The Cupz model of demyelination was used to evaluate the role for TACE in OPS during remyelination. $\boldsymbol{A}$, Timeline depicts the experimental design and time points of analysis (arrows). Adult 8- to 12-week-old PDGFR $\alpha$-Cre ${ }^{\mathrm{tm}}: \mathrm{TACE}^{\mathrm{w} / \mathrm{w}}: \mathrm{Rosa}^{\mathrm{Y}} \mathrm{YFP}$ (w/w) and PDGFR $\alpha$-Cre ${ }^{\mathrm{tm}}$ :

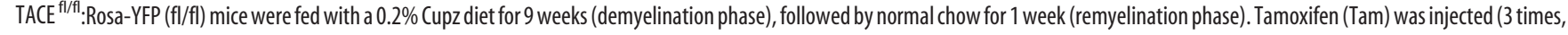
$24 \mathrm{~h}$ apart) after (upz removal, and brains were analyzed at 1 or 2 weeks later. Western blot $(\boldsymbol{B}, \boldsymbol{C})$ and immunofluorescence $(\boldsymbol{D})$ analysis of myelin-related protein levels at 1 and 2 weeks of recovery revealed a delay in SCWM remyelination in fl/fl mice. C, Histogram shows the quantification of optical density (0.D.) in $\boldsymbol{B}$, expressed in arbitrary units (a.u) after $\beta$-actin normalization. $\boldsymbol{E}$, Electron microcopy analysis of the SCWM at 1 week after last Tam injection in fl/fl and w/w mice. $\boldsymbol{F}-\boldsymbol{L}$, SCWM tissue sections were used to characterize the different stages of the oligodendroglial cell population in PDGFR $\alpha-\mathrm{Cre}^{\mathrm{tm}}$ :TACE ${ }^{\mathrm{w} / \mathrm{w}}$ :Rosa-YFP (w/w) and PDGFR $\alpha$-Cre ${ }^{\mathrm{tm}}:$ TACE $^{\mathrm{fl} / \mathrm{fl}}$ :Rosa-YFP (fl/fl) mice at 1 week after last Tam. $\boldsymbol{F}, \boldsymbol{H}$, Quantitative analysis of $\mathrm{CC} 1^{+}$cell numbers in the SCWM at 1 week after last tamoxifen injection. $\boldsymbol{F}, \mathbf{G}$, Histograms represent the percentage of $\mathrm{YFP}^{+}$cells that were either $\mathrm{CC}^{+}(\mathrm{OLs})$ or NG2 ${ }^{+}(\mathrm{OPS})$. Quantification of OP proliferation $\left(\mathrm{BrdU}{ }^{+} / \mathrm{NG2}^{+} ; \boldsymbol{F}, \boldsymbol{I}\right)$ and cell death (Caspase $3^{+}$cells; $\left.\boldsymbol{F}, \boldsymbol{J}\right)$ in the SCWM at 1 week of recovery. $\boldsymbol{K}-\boldsymbol{N}$, Adult NG2-Cre:TACE ${ }^{\text {fl/fl }}$, EGFR ${ }^{\text {Wa2/Wa2 }}$, and their corresponding control mice (NG2-Cre:TACE ${ }^{\text {w/w }}$ and EGFR ${ }^{\text {w/w }}$, respectively) were fed with cuprizone for 9 weeks followed by regular diet for 1 week. WB $(K, L)$ and immunofluorescence $(\boldsymbol{N})$ analysis of pEGFR and myelin related protein levels in the SCWM at 1 week of recovery in NG2-Cre:TACE ${ }^{\text {fl/f }}$ and EGFR ${ }^{\text {Wa2 } / \text { Wa2 }}$ mice. Data are shown as mean \pm SEM. $n=8-10$ mice for each time point. Scale bars: $\boldsymbol{D}, 100 \mu \mathrm{m} ; \boldsymbol{F}, 5 \mu \mathrm{m} ; \boldsymbol{E}, 500 \mathrm{~nm} ; \boldsymbol{N}, 50 \mu \mathrm{m} .{ }^{*} p<0.05$; ${ }^{* *} p<0.01,{ }^{* * *} p<0.001$, versus their respective WT mice.

firmed a delay in CNS remyelination in $\mathrm{fl} / \mathrm{fl}$ mice, as thinner remyelinated axons were observed in the SCWM as compared with their w/w littermates (Fig. 2E).

\section{TACE deficiency in OPs after demyelination perturbs \\ $O L$ regeneration}

To address whether the defects in CNS remyelination observed after TACE depletion in OPs are due to alterations in OL regeneration, we characterized OL lineage cells in the SCWM of $P D G F R \alpha-\mathrm{Cre}^{\mathrm{tm}}: \mathrm{TACE}^{\mathrm{w} / \mathrm{w}}:$ Rosa-YFP and PDGFR $\alpha-\mathrm{Cre}^{\mathrm{tm}}: \mathrm{TACE}^{\mathrm{t} / \mathrm{ft}}:$ Rosa-YFP mice at different time points during the process of remyelination following Cupz-induced demyelination. IF analysis at 1 week of the recovery phase evidenced reduced numbers of mature OLs in the SCWM of fl/fl mice compared with w/w mice (Fig. 2F-H). Analysis of the recombinant $\mathrm{YFP}^{+}$cells at 1 week of recovery showed that TACE depletion in OPs induced premature OL differentiation, as we detected an increased percentage of $\mathrm{YFP}^{+}$cells expressing markers of pre-OLs $\left(\mathrm{CCl}^{+}\right)$as compared with w/w mice (Fig. $2 F, G$ ). Accordingly, we found reduced numbers of proliferating $\mathrm{OPs}\left(\mathrm{NG}_{2}^{+} \mathrm{BrdU}^{+}\right)$within the whole SCWM OL population and within the $\mathrm{YFP}^{+}$cells in the SCWM of $\mathrm{fl} / \mathrm{fl}$ mice (Fig. 2F, I; data not shown), suggesting that TACE modulates OP cell expansion and differentiation during remyelination. Moreover, we found that TACE regulates OL lineage cell survival during remyelination, as we detected increased numbers of Caspase $3^{+}$and Caspase ${ }^{+} / \mathrm{YFP}^{+}$cells, in the fl/fl SCWM as compared with w/w mice (Fig. $2 F, J$ ). Altogether, these results suggest an essential function for TACE in modulating OL regeneration and the efficiency of CNS remyelination.
EGFR or TACE deficiency in OL lineage cells leads to similar defects in CNS remyelination following demyelination

TACE-mediated release of EGFR ligands from the cell surface via ectodomain shedding is considered essential for EGFR activation in several cell types (Blobel, 2005; Weber et al., 2012). Thus, we compared the capacity of OPs to remyelinate the demyelinated SCWM after constitutive deletion of TACE in OPs (NG2-cre: $\mathrm{TACE}^{\mathrm{f} / \mathrm{f}}$ ) or in EGFR hypofunctional (Egfr ${ }^{\text {Wa2/Wa2}}$ ) mice. NG2cre:TACE ${ }^{\mathrm{fl} / \mathrm{fl}}$, Egfr ${ }^{\mathrm{Wa} 2 / \mathrm{Wa} 2}$, and their corresponding control $(\mathrm{w} / \mathrm{w})$ littermates were fed with Cupz for 9 weeks as above. At 1 week after Cupz removal (recovery phase), we observed a similar delay in the remyelination process of both NG2:cre:TACE ${ }^{\mathrm{fl} / \mathrm{fl}}$ and Egfr ${ }^{\mathrm{Wa} 2 / \mathrm{Wa} 2}$ mice, as we detected a similar reduction in myelin related proteins levels in the SCWM compared with their control littermates, NG2-cre:TACE ${ }^{\mathrm{w} / \mathrm{w}}$ and $\mathrm{Egfr}^{\mathrm{w} / \mathrm{w}}$, respectively (Fig. $2 K, L)$. Moreover, $\mathrm{WB}$ and IF analysis at this time point revealed reduced EGFR activation levels in the SCWM of NG2-cre:TACE ${ }^{\mathrm{f} / \mathrm{fl}}$ mice as compared with $N G 2$-cre:TACE ${ }^{\mathrm{w} / \mathrm{w}}$ mice (Fig. $2 M, N$ ), suggesting a role for TACE in modulating EGFR activation in OL lineage cells during remyelination.

EGFR overexpression in TACE deficient OLs in vivo restores $O L$ development and SCWM myelination during postnatal

development

We recently demonstrated that EGFR overexpression in TACE ${ }^{\mathrm{f} / \mathrm{fl}}$ OPs in vitro restores the defects in OL cell survival and differentiation (Palazuelos et al., 2014). However, the relevance of EGFR in mediating TACE functions during OL development in vivo, at postnatal ages or during OL regeneration and CNS remyelination, has never been explored. To address this, we generated a new mouse line by crossing the NG2-cre:TACE ${ }^{\mathrm{f} / \mathrm{ft}}:$ Rosa-YFP 
A
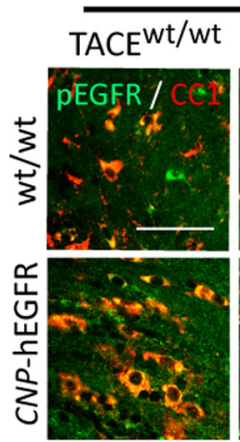

D

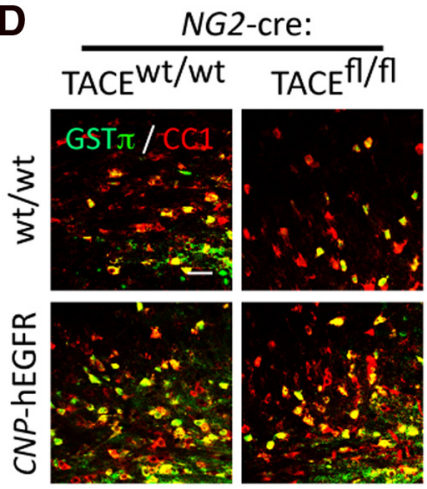

F
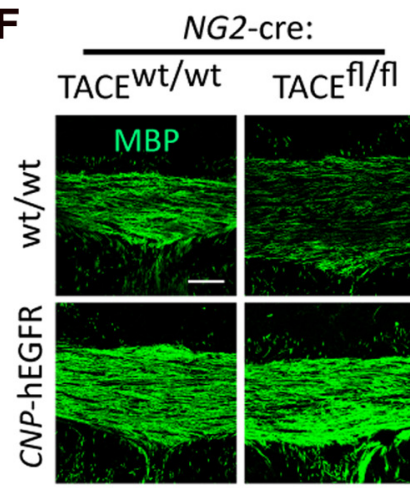

B

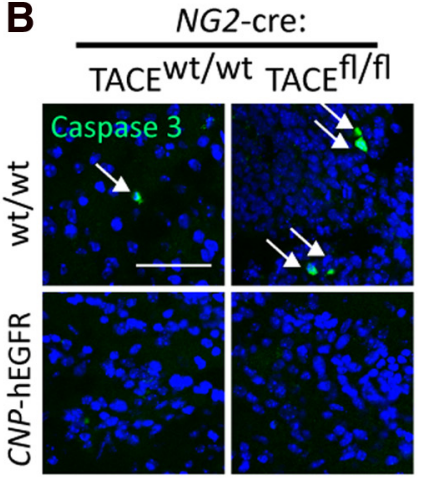

E
C

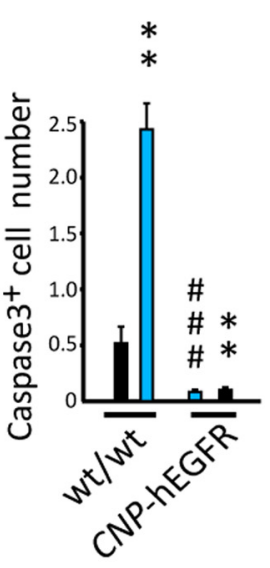

G
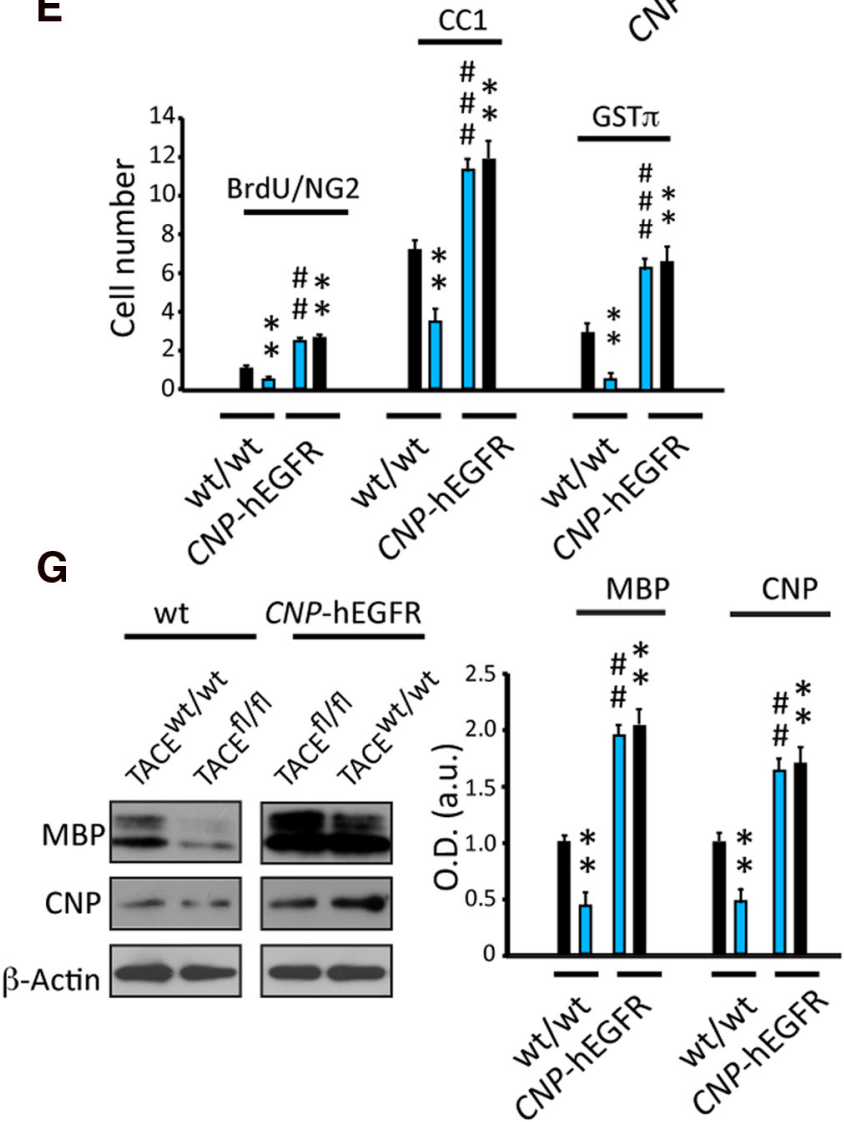

Figure 3. EGFR overexpression in TACE deficient OPS overcomes the defects in oligodendrogenesis and SCWM myelination during postnatal development. $A$, Immunofluorescence analysis of EGFR activation levels (pEGFR) in oligodendroglial cells $\left(C \mathrm{CC}^{+}\right)$at P15 in the SCWM of NG2-cre:TACE ${ }^{\text {fl/fl: }}$ :NP-hEGFR mice. $\boldsymbol{B}-\boldsymbol{E}$, Immunofluorescence analysis and quantification of caspase ${ }^{+}$cells $(\boldsymbol{B}, \boldsymbol{C})$, proliferating $\mathrm{OPs}\left(\mathrm{NG2}^{+} \mathrm{BrdU}^{+} ; \boldsymbol{E}\right)$, pre-OLs $\left(\mathrm{CC}^{+} ; \boldsymbol{D}, \boldsymbol{E}\right)$, and mature myelinating $\mathrm{OLS}\left(\mathrm{GST} \pi^{+} ; \boldsymbol{D}, \boldsymbol{E}\right)$ in the SCWM at P15. $\boldsymbol{F}$, Immunofluorescence analysis of MBP expression in the SCWM of NG2-cre:TACE ${ }^{\mathrm{fl} / \mathrm{fl}}:$ :NP-hEGFR mice and their littermates at P15. G, Western blot analysis and quantification of myelin-related proteins levels in the SCWM of NG2-Cre:TACE ${ }^{\mathrm{f} / \mathrm{fl}:}$ :CNP-EGFR mice and their littermates at P15. Data are shown as mean \pm SEM. $n=6-8$ brains for each genotype. Cell numbers are expressed as the number of positive cells per mm ${ }^{3}(\times 1000) . S c a l e ~ b a r s: A, B, D, 20$ $\mu \mathrm{m} ; \boldsymbol{F}, 100 \mu \mathrm{m} .{ }^{* *} p<0.01$ versus NG2-cre:TACE ${ }^{\mathrm{w} / \mathrm{w} ;}{ }^{\# \#} p<0.01,{ }^{\# \# \#} p<0.001$ versus NG2-cre:TACE ${ }^{\mathrm{fl} / \mathrm{fl}}$ mice.

mouse line with the CNP-hEGFR mouse line, in which the human EGFR gene is preferentially overexpressed in OL lineage cells under the control of the CNP promoter, (Aguirre et al., 2007), generating the NG2-cre:TACE ${ }^{\mathrm{fl} / \mathrm{fl}}: C N P$-hEGFR:Rosa-YFP mouse line. EGFR overexpression confers an enhanced basal activity without the need for ligand binding. We first analyzed oligodendrogenesis and SCWM myelination during the critical periods of postnatal development in this mouse line. As observed in the PDGFR $\alpha$-cre:TACE ${ }^{\mathrm{fl} / \mathrm{fl}}$ mouse line, we found reduced EGFR activation levels in OL lineage cells in the SCWM of NG2-cre:

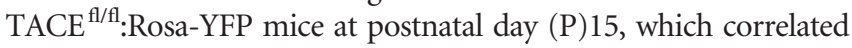
with increased OL lineage cell death, and defects in OP expansion,
OL development (Fig. 3A-E), and SCWM myelination (Fig. $3 F, G$ ). Next, we analyzed whether EGFR overexpression in OLs in vivo would rescue the defects in oligodendrogenesis and myelination observed in NG2-Cre:TACE ${ }^{\mathrm{fl} / \mathrm{fl}}$ mice. Interestingly, EGFR overexpression restored EGFR activation levels (Fig. 3A) and suppressed all of the cellular defects observed in NG2-cre:TACE ${ }^{\mathrm{fl} / \mathrm{fl}}$ mice during postnatal SCWM myelination, as increased OL cell survival and higher numbers of proliferating OPs and mature OLs were observed in the SCWM of NG2-cre:TACE ${ }^{\mathrm{fl} / \mathrm{fl}}: C N P$ hEGFR mice compared with NG2-cre:TACE ${ }^{\mathrm{fl} / \mathrm{fl}}$ mice (Fig. $3 B-E$ ). Finally, we analyzed SCWM myelination in these mouse lines. IF and WB analyses at P15 evidenced that EGFR overexpression in 
A

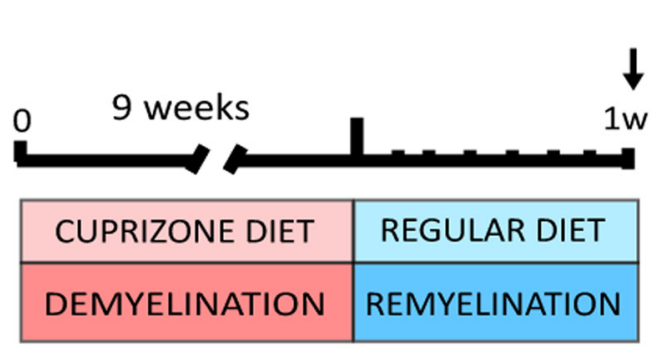

B

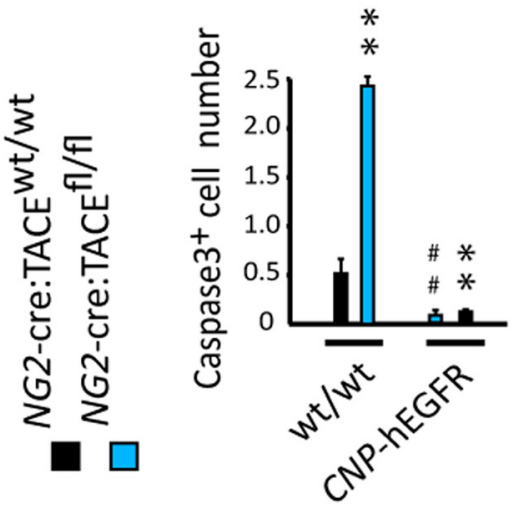

C

E

NG2-cre:
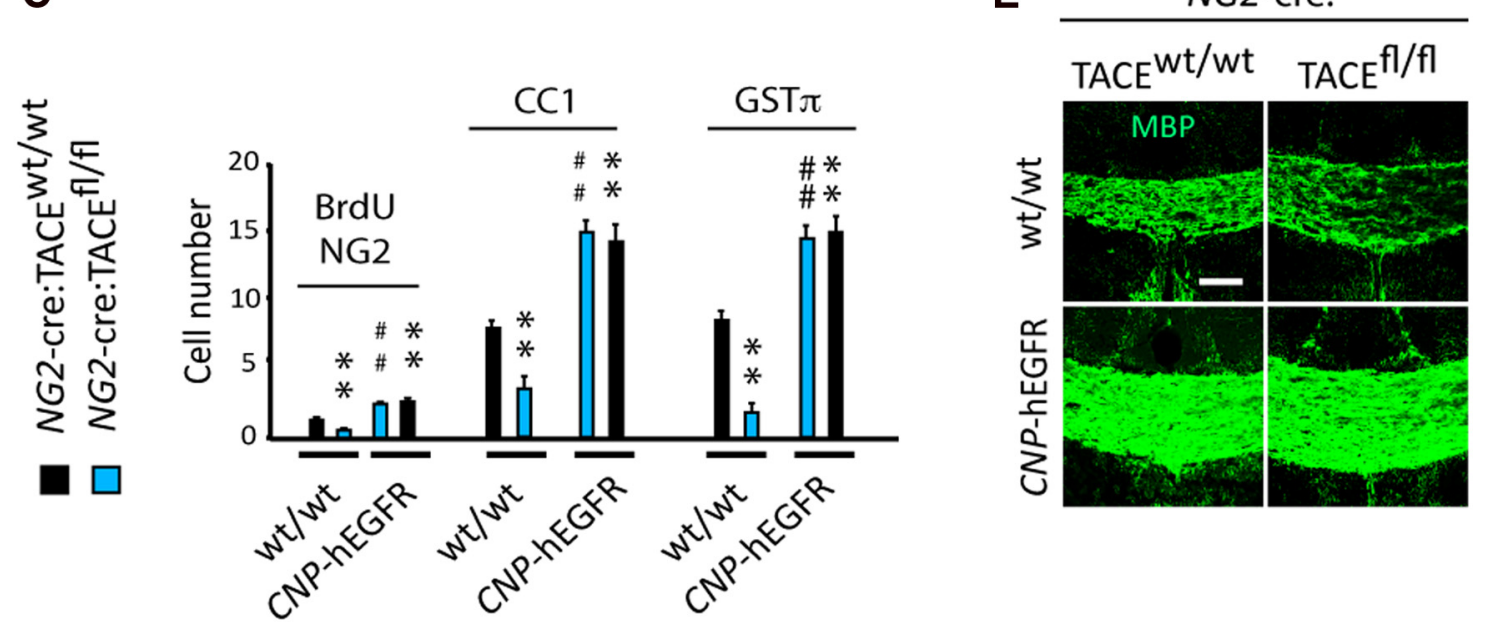

D
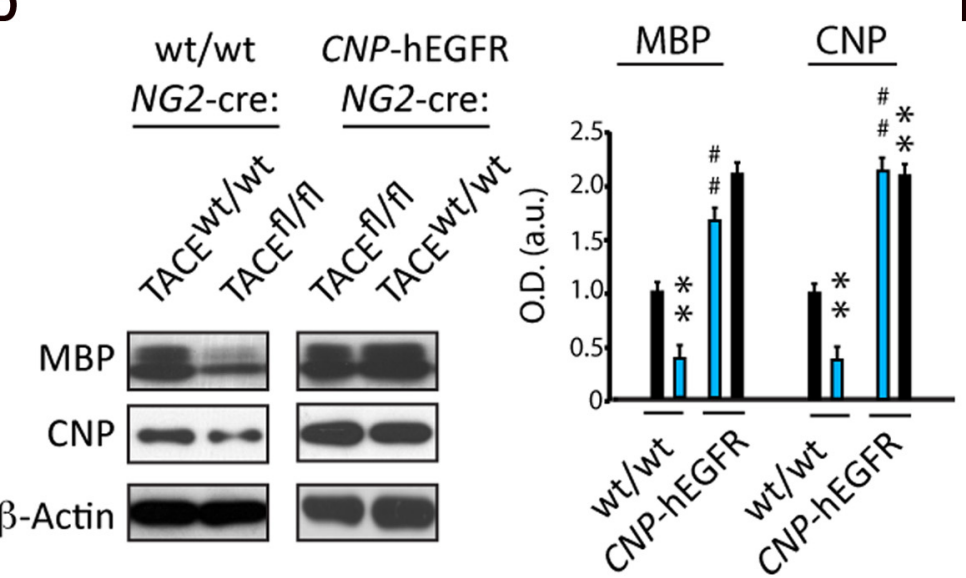

F
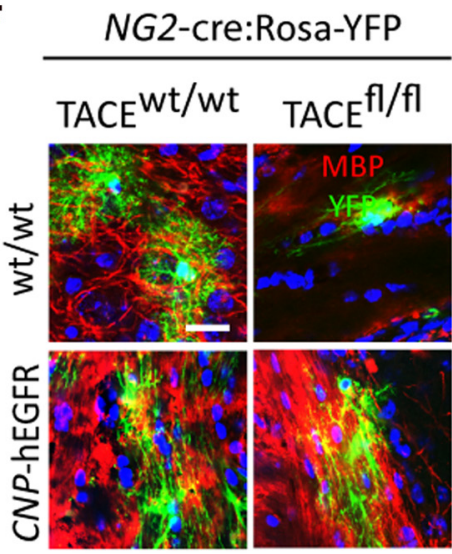

Figure 4. EGFR overexpression restores $0 \mathrm{~L}$ regeneration and CNS remyelination following demyelination in TACE ${ }^{\text {fl/fl }}$ mice. $A$, Adult NG2-cre:TACE ${ }^{\text {fl/fl }}$ :CNP-hEGFR:Rosa-YFP mice and their control littermates were fed cuprizone for 9 weeks and SCWM tissue was processed for histology and molecular analysis at 1 week after Cupz removal. Immunofluorescence analysis and quantification of caspase ${ }^{+}$cells $(\boldsymbol{B})$, proliferating OPs (NG2 ${ }^{+} \mathrm{BrdU}^{+}$cells; $\left.\boldsymbol{D}\right)$, pre-OLs (CC1 ${ }^{+}$cells; $\boldsymbol{C}$ ), and mature myelinating 0Ls (GST $\left.\pi^{+}, \mathrm{MBP} ; \boldsymbol{C}, \boldsymbol{D}, \boldsymbol{F}\right)$ in the SCWM of NG2-cre:TACE ${ }^{\mathrm{fl} / \mathrm{l}}$ :CNP-hEGFR:Rosa-YFP mice at 1 week after recovery. WB $(\boldsymbol{D})$ and IF $(\boldsymbol{E}, \boldsymbol{F})$ analysis and quantification of myelin related protein levels at 1 week after recovery. EGFR overexpression rescues the delay in $S C W M$ remyelination observed in TACE ${ }^{\mathrm{fl} / \mathrm{f}}$ mice. Data are shown as mean \pm SEM. $n=6-8$ brains for each genotype. Cell numbers are expressed as the number of positive cells per cubic millimeter $(X 1000)$. Scale bars: $E, 100 \mu \mathrm{m} ; \boldsymbol{F}, 10 \mu \mathrm{m} .{ }^{* *} p<0.01$ versus NG2-cre:TACE ${ }^{\mathrm{w} / \mathrm{w}}$ mice; \#\#p $<0.01$ versus NG2-cre:TACE ${ }^{\mathrm{fl} / \mathrm{fl}}$ mice.

TACE deficient OPs restored the defects in SCWM myelination, as similar myelin related protein levels were observed in the SCWM of NG2-cre:TACE ${ }^{\mathrm{f} / \mathrm{fl}}$ and NG2-cre:TACE ${ }^{\mathrm{w} / \mathrm{w}}$ mice in which EGFR was overexpressed (Fig. $3 F, G$ ). Altogether, these results indicate that TACE signals, at least in part, through EGFR to modulate OL survival and development, and subsequently, to ensure CNS myelination during postnatal development.
EGFR overexpression in TACE deficient OL lineage cells in vivo overrides the defects in OL regeneration and SCWM remyelination To confirm the above results in the context of remyelination, and to address the relevance of EGFR in mediating TACE functions in OL lineage cells under demyelinating conditions, we analyzed the regenerative capacity of NG2-cre:TACE ${ }^{\mathrm{f} / \mathrm{f}}:$ :CNP-hEGFR OPs after Cupz-induced demyelination. NG2-cre:TACE ${ }^{\mathrm{f} / \mathrm{f} l}:$ CNP-hEGFR 
mice and their littermates were fed with Cupz for 9 weeks and OL regeneration and remyelination was analyzed at 1 week after Cupz removal (Fig. 4A). Reduced OL lineage cell survival, OP proliferation, and OL regeneration was observed in NG2-cre: $\mathrm{TACE}^{\mathrm{f} / \mathrm{fl}}$ mice compared with NG2-cre:TACE ${ }^{\mathrm{w} / \mathrm{w}}$ mice (Fig. $4 B, C$ ). Interestingly, we observed that EGFR overexpression restored the defects in OL regeneration in the SCWM of TACE deficient OPs, as we found similar numbers of Caspase $3^{+}$, proliferating $\mathrm{OPs}\left(\mathrm{BrdU}^{+} / \mathrm{NG}^{+}\right)$and mature $\mathrm{OLs}\left(\mathrm{CC}^{+}\right.$, GST $\pi^{+}$) in the SCWM of CNP-hEGFR overexpressing NG2-cre: $\mathrm{TACE}^{\mathrm{w} / \mathrm{w}}$ and NG2-cre:TACE ${ }^{\mathrm{fl} / \mathrm{fl}}$ mice (Fig. $4 B, C$ ). IF and WB analysis in the SCWM at 1 week of Cupz removal evidenced that the observed defects in SCWM remyelination in NG2-cre:TA$\mathrm{CE}^{\mathrm{f} / / \mathrm{fl}}$ mice were rescued after EGFR overexpression, as similar levels of myelin proteins were found in the SCWM of w/w and fl/fl EGFR-overexpressing mice (Fig. $4 D-F$ ).

\section{Discussion}

TACE has been linked to numerous developmental processes, but more importantly, to pathological conditions, such as inflammation, cancer, and neurodegeneration (Scheller et al., 2011). In the context of neuroinflammation and demyelinating disorders, such as MS, the use of TACE inhibitors has been proposed for reducing inflammation to prevent further neurodegeneration (Rose-John, 2013). However, in the present study, we provide evidence of an essential function for TACE in modulating the process of oligodendrogenesis in the context of OL regeneration and CNS remyelination. Therefore, pharmacological approaches targeting TACE inhibition may blunt the process of OP expansion and OL regeneration, and delay the process of CNS remyelination, suggesting that TACE manipulation under demyelinating conditions may produce opposite outcomes by acting on immune and OL lineage cells. Indeed, increased TACE levels have been detected in peripheral blood mononuclear cells of SPMS and RRMS patients during clinical remission as compared with healthy controls and PPMS patients (Comabella et al., 2006). Further, alterations in TACE levels have been associated with lesion activity in MS patients (Weinger et al., 2009), suggesting a role for TACE in MS progression. Importantly, our study describes the presence of TACE in OL lineage cells in the NAWM of MS patients, and suggests that TACE activation may promote OL regeneration and CNS remyelination. Moreover, our study shows that TACE deficiency in OL lineage cells reduces EGFR activation levels in the SCWM after demyelination, and that EGFR overexpression appears to be sufficient to rescue the defects in SCWM myelination and remyelination of TACE deficient OLs. However, due to the numerous targets described for TACE in other cell types, we cannot exclude the possibility that TACE may have other substrates in OL lineage cells, and therefore, modulate other signaling pathways essential during oligodendrogenesis in health and disease. Overall, this study opens the possibility of manipulating TACE activity in the design of new therapeutic strategies in demyelinating disorders by promoting $\mathrm{OL}$ regeneration and myelin repair.

\section{References}

Aguirre A, Gallo V (2007) Reduced EGFR signaling in progenitor cells of the adult subventricular zone attenuates oligodendrogenesis after demyelination. Neuron Glia Biol 3:209-220. CrossRef Medline
Aguirre A, Dupree JL, Mangin JM, Gallo V (2007) A functional role for EGFR signaling in myelination and remyelination. Nat Neurosci 10:9901002. CrossRef Medline

Blobel CP (2005) ADAMs: key components in EGFR signalling and development. Nat Rev Mol Cell Biol 6:32-43. CrossRef Medline

Comabella M, Romera C, Camiña M, Perkal H, Moro MA, Leza JC, Lizasoain I, Castillo M, Montalban X (2006) TNF-alpha converting enzyme (TACE) protein expression in different clinical subtypes of multiple sclerosis. J Neurol 253:701-706. CrossRef Medline

Edwards DR, Handsley MM, Pennington CJ (2008) The ADAM metalloproteinases. Mol Aspects Med 29:258-289. CrossRef Medline

Fancy SP, Chan JR, Baranzini SE, Franklin RJ, Rowitch DH (2011) Myelin regeneration: a recapitulation of development? Annu Rev Neurosci 34: 21-43. CrossRef Medline

Huang JK, Fancy SP, Zhao C, Rowitch DH, Ffrench-Constant C, Franklin RJ (2011) Myelin regeneration in multiple sclerosis: targeting endogenous stem cells. Neurotherapeutics 8:650-658. CrossRef Medline

Ivkovic S, Canoll P, Goldman JE (2008) Constitutive EGFR signaling in oligodendrocyte progenitors leads to diffuse hyperplasia in postnatal white matter. J Neurosci 28:914-922. CrossRef Medline

Klingener M, Chavali M, Singh J, McMillan N, Coomes A, Dempsey PJ, Chen EI, Aguirre A (2014) N-cadherin promotes recruitment and migration of neural progenitor cells from the SVZ neural stem cell niche into demyelinated lesions. J Neurosci 34:9590-9606. CrossRef Medline

Luetteke NC, Phillips HK, Qiu TH, Copeland NG, Earp HS, Jenkins NA, Lee DC (1994) The mouse waved-2 phenotype results from a point mutation in the EGF receptor tyrosine kinase. Genes Dev 8:399-413. CrossRef Medline

Palazuelos J, Crawford HC, Klingener M, Sun B, Karelis J, Raines EW, Aguirre A (2014) TACE/ADAM17 is essential for oligodendrocyte development and CNS myelination. J Neurosci 34:11884-11896. CrossRef Medline

Peschon JJ, Slack JL, Reddy P, Stocking KL, Sunnarborg SW, Lee DC, Russell WE, Castner BJ, Johnson RS, Fitzner JN, Boyce RW, Nelson N, Kozlosky CJ, Wolfson MF, Rauch CT, Cerretti DP, Paxton RJ, March CJ, Black RA (1998) An essential role for ectodomain shedding in mammalian development. Science 282:1281-1284. CrossRef Medline

Pruessmeyer J, Ludwig A (2009) The good, the bad and the ugly substrates for ADAM10 and ADAM17 in brain pathology, inflammation and cancer. Semin Cell Dev Biol 20:164-174. CrossRef Medline

Rivers LE, Young KM, Rizzi M, Jamen F, Psachoulia K, Wade A, Kessaris N, Richardson WD (2008) PDGFRA/NG2 glia generate myelinating oligodendrocytes and piriform projection neurons in adult mice. Nat Neurosci 11:1392-1401. CrossRef Medline

Rose-John S (2013) ADAM17, shedding, TACE as therapeutic targets. Pharmacol Res 71:19-22. CrossRef Medline

Scafidi J, Hammond TR, Scafidi S, Ritter J, Jablonska B, Roncal M, SzigetiBuck K, Coman D, Huang Y, McCarter RJ Jr, Hyder F, Horvath TL, Gallo $\mathrm{V}$ (2014) Intranasal epidermal growth factor treatment rescues neonatal brain injury. Nature 506:230-234. CrossRef Medline

Scheller J, Chalaris A, Garbers C, Rose-John S (2011) ADAM17: a molecular switch to control inflammation and tissue regeneration. Trends Immunol 32:380-387. CrossRef Medline

Weber S, Saftig P (2012) Ectodomain shedding and ADAMs in development. Development 139:3693-3709. CrossRef Medline

Weinger JG, Omari KM, Marsden K, Raine CS, Shafit-Zagardo B (2009) Up-regulation of soluble Axl and Mer receptor tyrosine kinases negatively correlates with Gas6 in established multiple sclerosis lesions. Am J Pathol 175:283-293. CrossRef Medline

Yang P, Baker KA, Hagg T (2006) The ADAMs family: coordinators of nervous system development, plasticity and repair. Prog Neurobiol 79:73-94. CrossRef Medline

Zhu X, Bergles DE, Nishiyama A (2008) NG2 cells generate both oligodendrocytes and gray matter astrocytes. Development 135:145-157. Medline 